\section{A Semi-Automated Algorithm for Segmentation of the Left Atrial Appendage Landing Zone: Application in Left Atrial Appendage Occlusion Procedures}

\author{
Pakizeh Moghadam A. ${ }^{1 \oplus}$, Eskandari M.., Monaghan M. J.3, \\ Haddadnia J.4*(1)
}

\begin{abstract}
Background: Mechanical occlusion of the Left atrial appendage (LAA) using a purpose-built device has emerged as an effective prophylactic treatment in patients with atrial fibrillation at risk of stroke and a contraindication for anticoagulation. A crucial step in procedural planning is the choice of the device size. This is currently based on the manual analysis of the "Device Landing Zone" from echocardiographic images.
\end{abstract}

Objective: We aimed to develop an algorithm for automated segmentation of the LAA landing zone from 3D echocardiographic images of the LAA.

Material and Methods: In this experimental study, 2D axial images were derived from the $3 \mathrm{D}$ echo datasets. After image pre-processing, binary images were created using a thresholding method. A binary image matrix was then formed and scanned using 8-adgacency approach resulting in segmentation of the objects with a closed circumference within the image. Erosion/dilation techniques were then applied to remove small objects. A feature-based approach was then used to firstly detect the LAA region and secondly to identify the device landing zone.

Results: A total of 22 datasets were used in this study. The algorithm produced up to 9 axial images as the proposed landing zone. The selected axial images were compared to the echocardiographic images. In 18 cases $(81.8 \%)$, the algorithm successfully segmented the LAA and proposed the landing zone based on the defined features.

Conclusion: We have developed a simple and fast algorithm for semi-automated segmentation of the LAA landing zone. Further studies are needed to assess the accuracy of the proposed landing zones by this method.

Citation: Pakizeh Moghadam A, Eskandari M, Monaghan MJ, Haddadnia J. A Semi-Automated Algorithm for Segmentation of the Left Atrial Appendage Landing Zone: Application in Left Atrial Appendage Occlusion Procedures J Biomed Phys Eng. 2020;10(2):205-214. (661/jbe.vi0.1912-1019.

Keywords

Atrial Appendage; Atrial Fibrillation; Imaging, Three-Dimensional

\section{Introduction}

troke is the leading cause of long-term disability and the second most common cause of mortality. Cardio-embolic stroke accounts for up to $30 \%$ of cases and often is associated with the most severe
${ }^{1} \mathrm{PhD}$ candidate, Department of Electrical and Computer Engineering, Hakim Sabzevari Univer-

sity, Sabzevar, Iran

${ }^{2} \mathrm{MD}$, Department of Car-

diology, King's College

Hospital, London, UK

${ }^{3} \mathrm{PhD}$, Department of

Cardiology, King's Col-

lege Hospital, London,

UK

${ }^{4} \mathrm{PhD}$, Department of

Electrical and Computer

Engineering, Hakim

Sabzevari University,

Sabzevar, Iran

Corresponding author:

J. Haddadnia

Department of Electrical and Computer Engineer

ing, Hakim Sabzevari

University, Sabzevar, Iran

E-mail: haddadnia@hsu. ac.ir

Received: 16 December 2019 Accepted: 26 December 2019 
forms of stroke. Atrial fibrillation (AF), is the main cause of cardio-embolic stroke through clot formation in the left atrial appendage (LAA) [1, 2]. The recommended treatment for prevention of stroke in those with non-valvular AF at high risk of stroke is lifelong anticoagulation [3]. However, anticoagulation is contraindicated in patients with previous lifethreatening bleeding and increases the risk of major bleeding in the often elderly cohort of patients with AF. Percutaneous, catheterbased, mechanical occlusion of the LAA has emerged as an effective treatment and is rapidly growing.

Meticulous procedural planning is crucial in securing a successful LAA occlusion procedure. The LAA is known to have significant anatomical variation between patients. An important step in procedural planning is analysis of the LAA Landing Zone, an anatomical plane below the ostium of the LAA, which is used for device type/size selection. In the early days, 2D echocardiography was the main modality used for procedural planning of LAA occlusion. It was soon noted that owing to the often elliptical shape of the landing zone, 2D echocardiography may fall short in accurate estimation of shortest and longest dimensions of the landing zone. This may lead to resizing during the procedure which will add to the cost of an already expensive procedure. Moreover, deployment of an undersized or oversized device may lead to dislodgment and embolization of the device, para-device leak or LAA rupture/injury to vital adjacent structures respectively. Therefore, a 3D imaging modality that allows interrogation of the LAA in different planes is strongly recommended for procedural planning of LAA occlusion procedures. 3D transesophageal echocardiography (TEE) is the preferred method recommended by expert consensus for this purpose [4].

Another recognized source of error in cardiac imaging that is pertinent to the assessment of the LAA landing zone is inter and intra observer variability resulting from the manual analysis of echo datasets. Focusing on developing automated models to minimize the user-image interaction for analysis of echocardiographic images has been an area of interest in imaging research. In this study, we aimed to develop an algorithm for automated segmentation/identification of the LAA landing zone from 3D echocardiographic images.

\section{Material and Methods}

The data used in this experimental study were 3D echocardiographic images of 22 selected patients who successfully underwent LAA closure at King's College Hospital, London, UK. The data were entirely anonymized and untraceable, and were used in accordance with European General Data Protection Regulation (GDPR) standards, ethical standards of the Hakim University, Sabzevar, Iran and with the 1964 Helsinki Declaration and its later amendments or comparable ethical standards. Images were obtained using an Epiq 7XT echocardiography machine using $\mathrm{X} 7$ or $\mathrm{X} 8 \mathrm{-2} \mathrm{t}$ probes (Philips, Best, Netherland) in a zoomed 3D mode.

As shown in Figure 1, the LAA landing zone is located in the axial (transverse) plane, in an area of the LAA that has the least variation in its perimeter (red box). We therefore aimed to develop an automated algorithm to segment the LAA and to identify the landing zone based on its morphological features. First, 3D zoomed datasets of the LAA were imported into an open source platform (Slicer), reviewed by an experienced echocardiographer who selected the best frame for analysis of the LAA. A series of 208 DICOM images of the selected 3D volume was exported from the slicer. The axial DICOM images converted to $208176 \times 208$ pixel images in JPEG format in MATLAB (Figure 2).

\section{Image Pre-processing}

Common issues in medical imaging include the noise resulting from receiving at high frequency, varying background illuminations, 
An Algorithm for LAA Segmentation
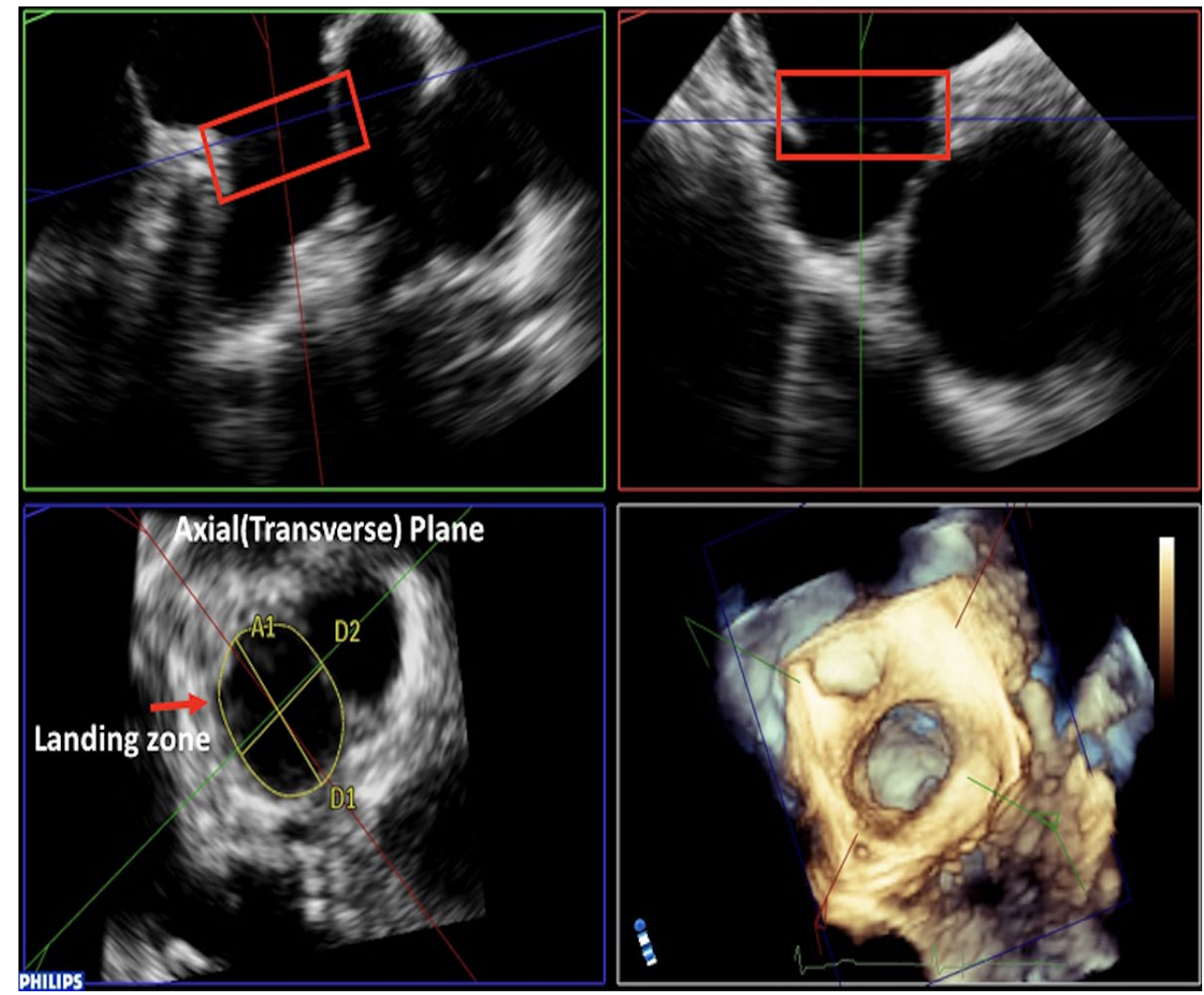

Figure 1: Identification of the left atrial appendage landing zone using multi-planar reconstruction of zoomed 3D echo of the appendage
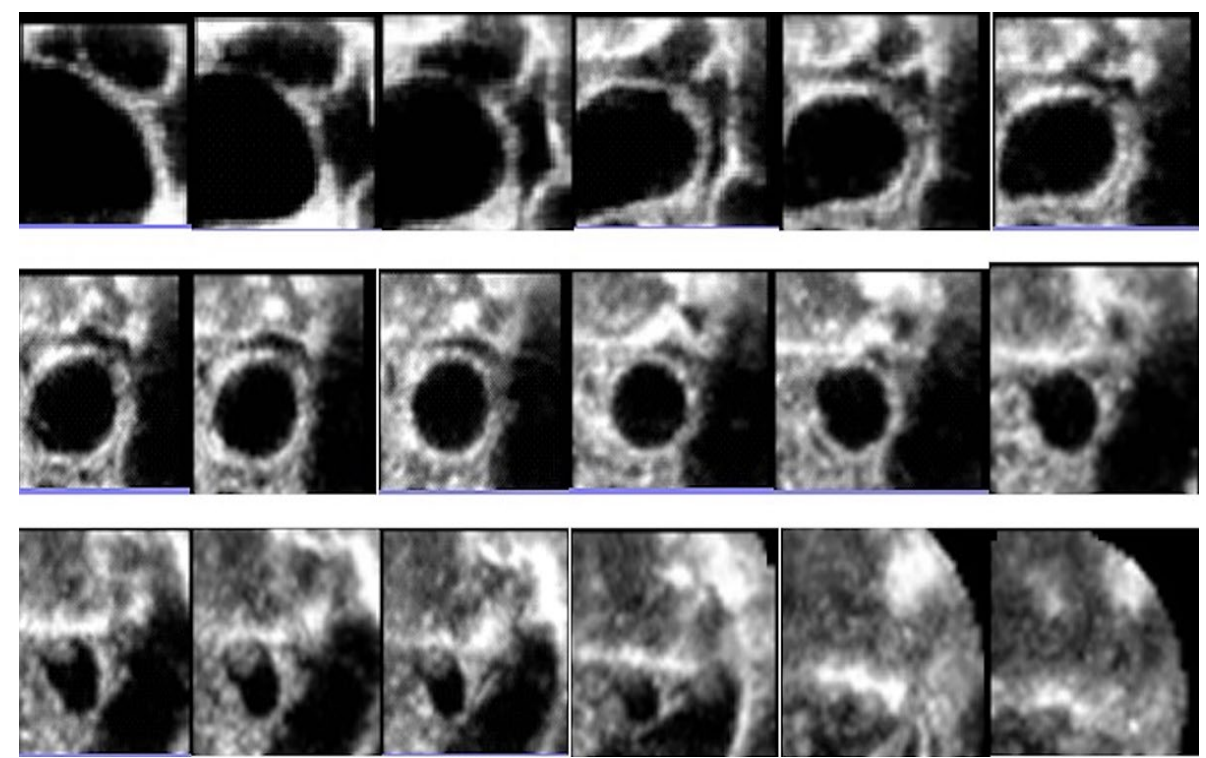

Figure 2: Example of converted axial DICOM images to jpeg format 
and distant structure orientation problems [5]. Therefore, image pre-processing was performed systematically on all 2D axial images as follows.

\section{Shifting Images}

Since each image has a black border of different size, the borders must be automatically removed in order to assimilate all images. For this purpose, pixels at the corner of the image with zero grayscale were removed, the size of remaining image was measured, and the point of comparison $(0,0)$ was shifted to the top left corner of the image.

\section{Smoothing Filters}

The Gaussian filter is a low-pass and linear filter that is used for reducing the image noise. Two, one-dimensional Gaussian filters can be used instead of one, two-dimensional kernel to speed up the convolution operation. This convolves the image once horizontally with a one-dimensional kernel, and then vertically [6]. The two-dimensional Gaussian filter is calculated using the following equation.

$$
K(i, j)=\frac{1}{2 \Pi \sigma} e^{-\frac{i+j}{2 \sigma}}, \sigma=\text { Variance }
$$

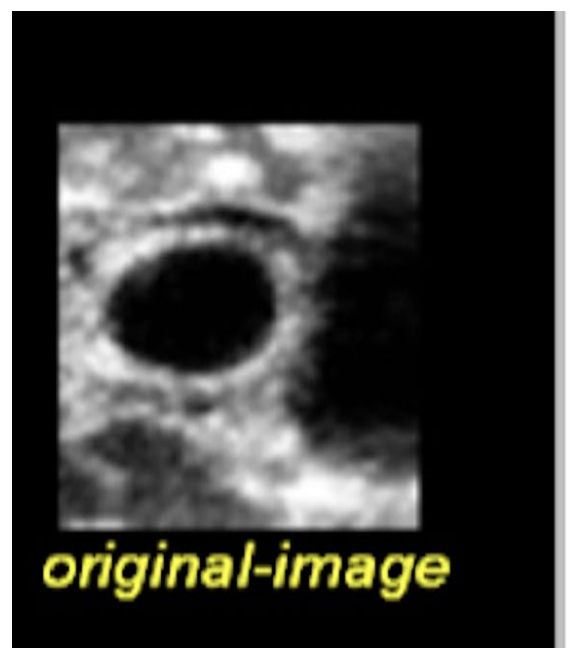

Window size should be large enough for Gaussian filtering. Increasing standard deviation creates more smoothing. This parameter $(\sigma)$ that sets the standard deviation for the function was considered 2 in this study. The effect of the Gaussian filter alone on the images is shown in Figure 3.

\section{Gamma Correction}

Today, many imaging tools leave non-linear changes in the brightness of image pixels due to technical limitations or the surrounding environment. Moreover, cameras fall significantly short in implementing the human eye capability of displaying an image texture and depth, resulting in some distortion of the image perception. Gamma correction is a nonlinear operation with $\gamma$ parameter.

If $\gamma<1$, the image will become brighter, if $\gamma>1$, the image will become darker, and if $\gamma=1$, no changes will be made to the image. One of the advantages of gamma correction is that absolute black and white points don't change. Gamma reduction can achieve a better representation of the images with darker pixels. In order to find a value for $\gamma$, the most common method is to move the histogram to the middle, so that very bright images become darker and darker images become brighter.

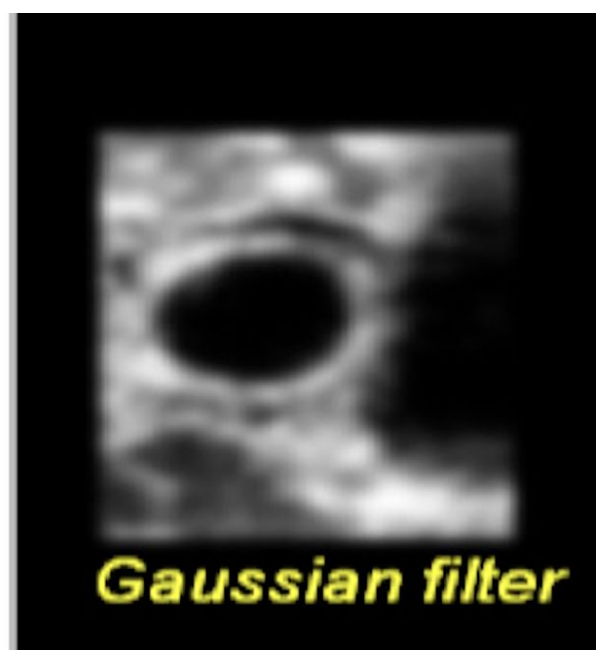

Figure 3: An example of the effect of Gaussian filter on an axial image of the LAA 
In order to move and adjust the histogram to the middle, one should have the length of number format representing image brightness. The number format often lacks the 8-bit sign. Since the goal is to move the histogram to the middle, the length of the number format is assumed to be L in general. Therefore, the average output image should be approximately equal to $\mathrm{L} / 2$. Gamma is obtained according to the following equation (2).

$$
\gamma=(\log |\mathrm{X}-\mathrm{L} / 2|)^{-\mathrm{i}}
$$

If the mean is higher than $128, \mathrm{i}=-1$, otherwise, it is assumed to be 1 . Considering the nature of available echocardiographic pictures, it is necessary to make them brighter $(\gamma<1)$. The gamma value used by the present study will therefore be 0.125 , according to the aforementioned points. The results of applying gamma corrections alone on a sample image is shown in Figure 4.

\section{Edge Improvement}

Edges are boundaries of objects within an image. Human perception is very sensitive to edges, and since edges are made of highfrequency components, image visual quality is significantly reduced in case of distortion by high-frequency components [7, 8]. At the same time, improving high-frequency components will increase image visual quality. Image sharpening techniques highlight the edges and details of the image. The median filter is used for sharpening the image. Filter output is a factor of the difference between the central pixel and its smallest neighbours. Therefore, the filter output is a big value for edges of the image and small for its flat parts. Although this method can show the edges of the images, it performs differently for positive and negative slope edges. The problem is, since output corresponds to the factor of difference between the central and the smallest pixel, it has the smallest value for negative slope edges, such as the central pixel, so the output value is small. Moreover, if the central pixel has the same value as the smallest neighbouring pixel, the filter output will be zero. This means that negative slope edges will not be extracted as well as positive ones. To overcome this limitation, the edge detection structure is changed according to Figure 5. Stages will be as follows:

- Positive slope edges are found using a high-pass filter.

- The signal is changed with pre-processing, which changes negative slope edges to posi-
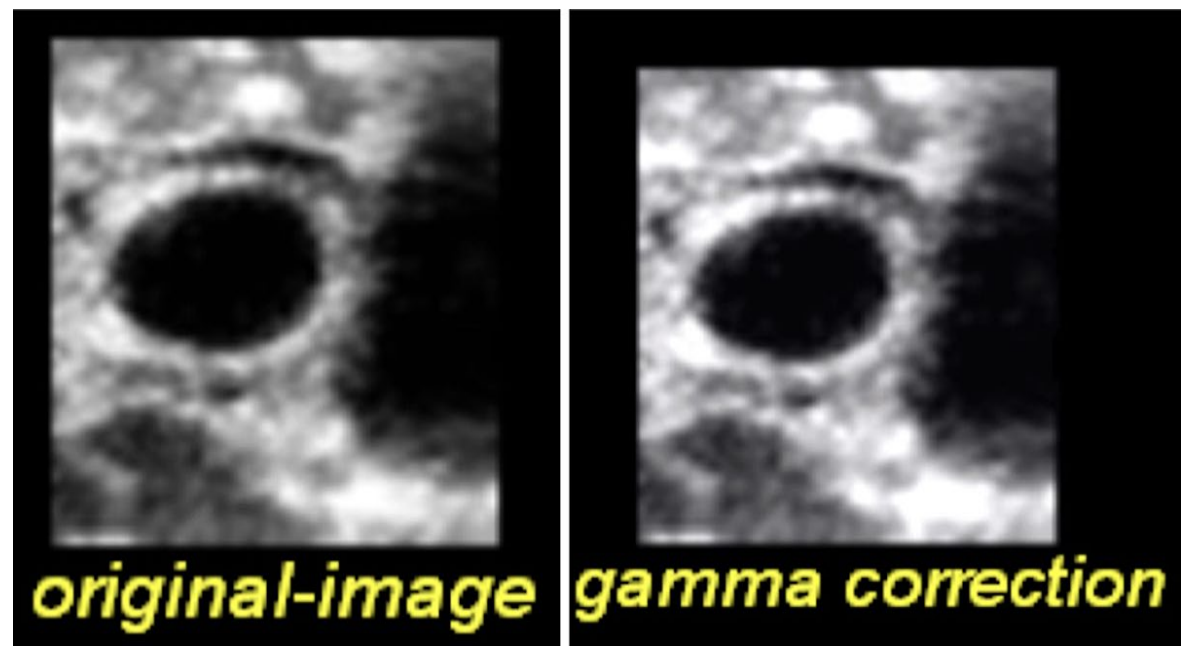

Figure 4: An example of effect of gamma correction on an axial image of the LAA 
tive slope ones, and a high-pass filter finds their slopes.

- The main signal is added by a factor of positive and negative edges.

These stages are shown in the diagram in Figure 5, the upper part of which detects positive slope edges, while the middle part finds negative ones. Applying modifications will improve edges (Figure 6).

\section{Segmentation and Image Analysis}

Thresholding

Thresholding is considered as one of commonly used methods for segmenting images

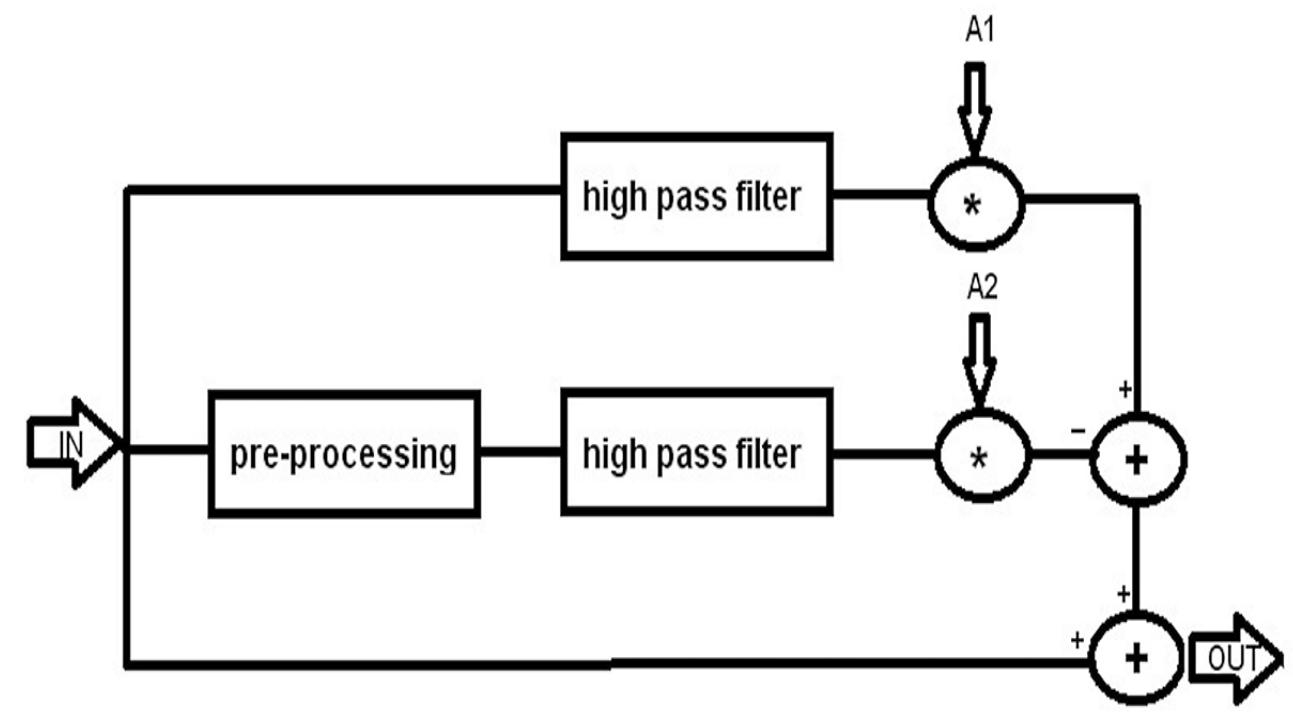

Figure 5: Steps of edge improvement on the axial images of the LAA
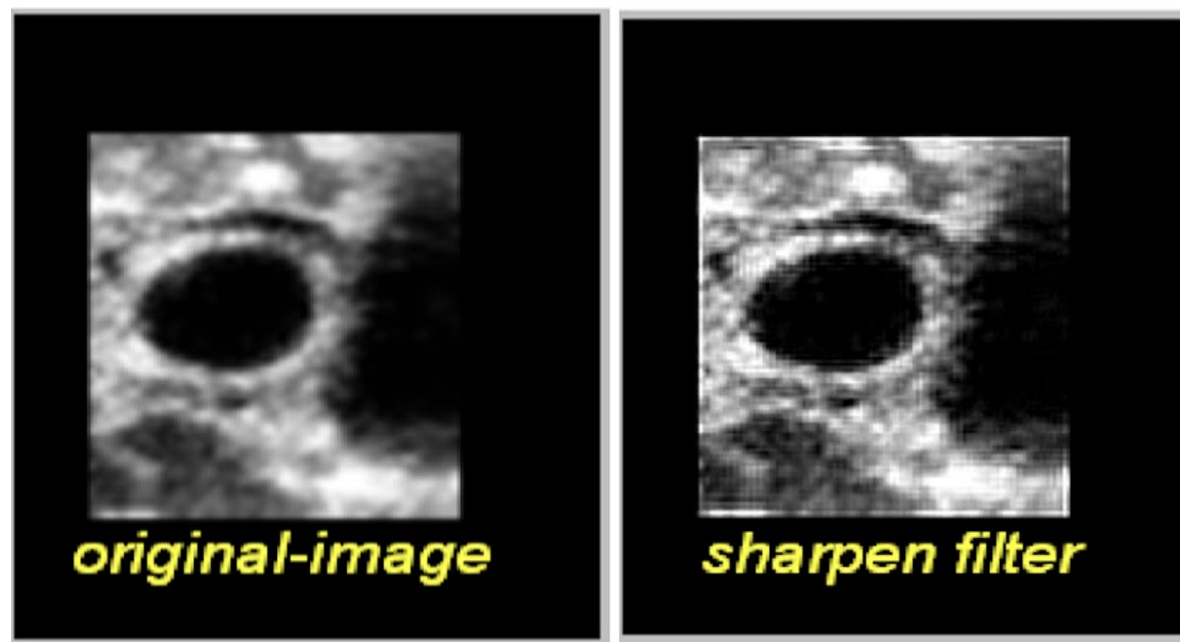

Figure 6: An example of the effect of edge improvement on an axial image of the LAA 
[9]. A binary image is obtained by applying a thresholding method on a grayscale image, where the boundary of objects within the image is detected with high accuracy. There are various methods for thresholding. The balanced thresholding method divides the image histogram in half and, similar to a scale, finds the heavier segment (right or left side). It then removes the last load histogram from the heavier segment. The histogram is divided in half once again and so forth until both sides of the scale reach each other. Based on this method, the threshold value for making binary images of the echo pictures used in this study, was 0.5 to 0.6 of the average brightness of pixels in each image [10].

In the next step, each binary image is first scanned and the values of each pixel, 0 or 1 , are stored in a binary image matrix. The image is then scanned from the beginning and the first pixel that has a value of 1 is selected. Pixels within the 8-adjacency are then scanned and those with a value of 1 are given a label. Thereafter, for each pixel found with a value of 1, the same 8-adjacency process and labelling is carried out until there is no pixel with a value of 1 left in the adjacency. Essentially, this method will result in segmenting hollow objects in the image with closed circumference and therefore as shown in Figure 7 (arrows) it may segment other objects in addition to the LAA region. The erosion/dilation techniques are then applied to remove some of the small segmented objects.

The next step was to identify the LAA landing zone from the segmented objects. The LAA cross sectional plane at the level of the landing zone is a circle or ellipse closed from all directions. As explained in the methods section we hypothesised that the variation in the perimeter of the thin axial images in the vicinity of the landing zone would be negligible.

Therefore, we used the following features to identify axial images that are expected to represent the landing zone:

- The LAA area in pixels

- The LAA perimeter in pixels

- Area to perimeter ratio: with a value between 0 and 1

- Longest dimension of the axial LAA image

- Shortest dimension of the axial LAA image as defined by a perpendicular line to the longest

- Object position
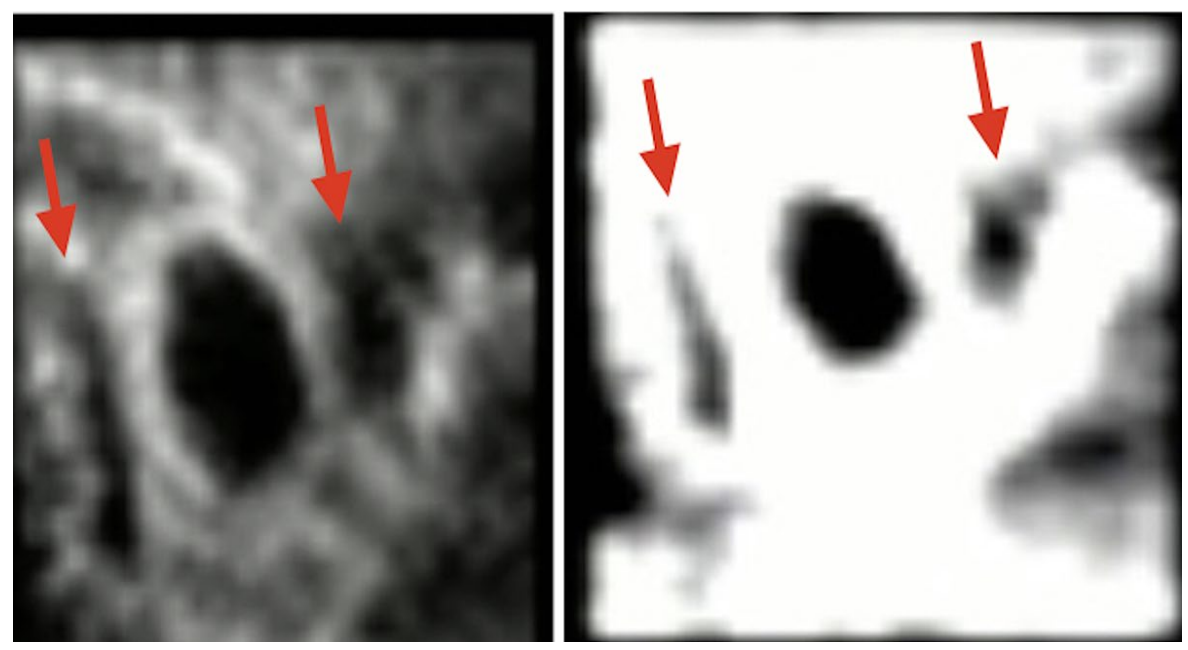

Figure 7: Axial image of the LAA contains other objects with closed circumference that will be segmented alongside with the LAA 
Using these features up to 9 axial images were extracted as the landing zone.

It is recognized that although image pre-processing optimizes the image quality, it might result in errors in the size of the object. Therefore, we repeated the segmentation process/ feature extraction on the original axial images without pre-processing. Another set of up to 9 images representing the landing zone was produced.

The two sets of results (from segmentation on original images and images with pre-processing) were compared and the comparable images in the both sets were chosen as the final images representing the LAA landing zone (Figure 8).

\section{Results}

A total of $223 \mathrm{D}$ echo datasets were used in this study. The proposed algorithm produced up to 9 axial images representing the proposed landing zone. The selected axial images were qualitatively compared to the echocardiographic images. In 18 cases $(81.8 \%)$, the algorithm successfully segmented the area of interest in the LAA.

Failure in creating binary images was identified as the culprit for 4 unsuccessful cases. The original axial images of these cases appeared to be very noisy particularly at the level of the LAA ostium. As such, image pre-processing (smoothing, gamma correction, edge detection) resulted in an average image grayscale

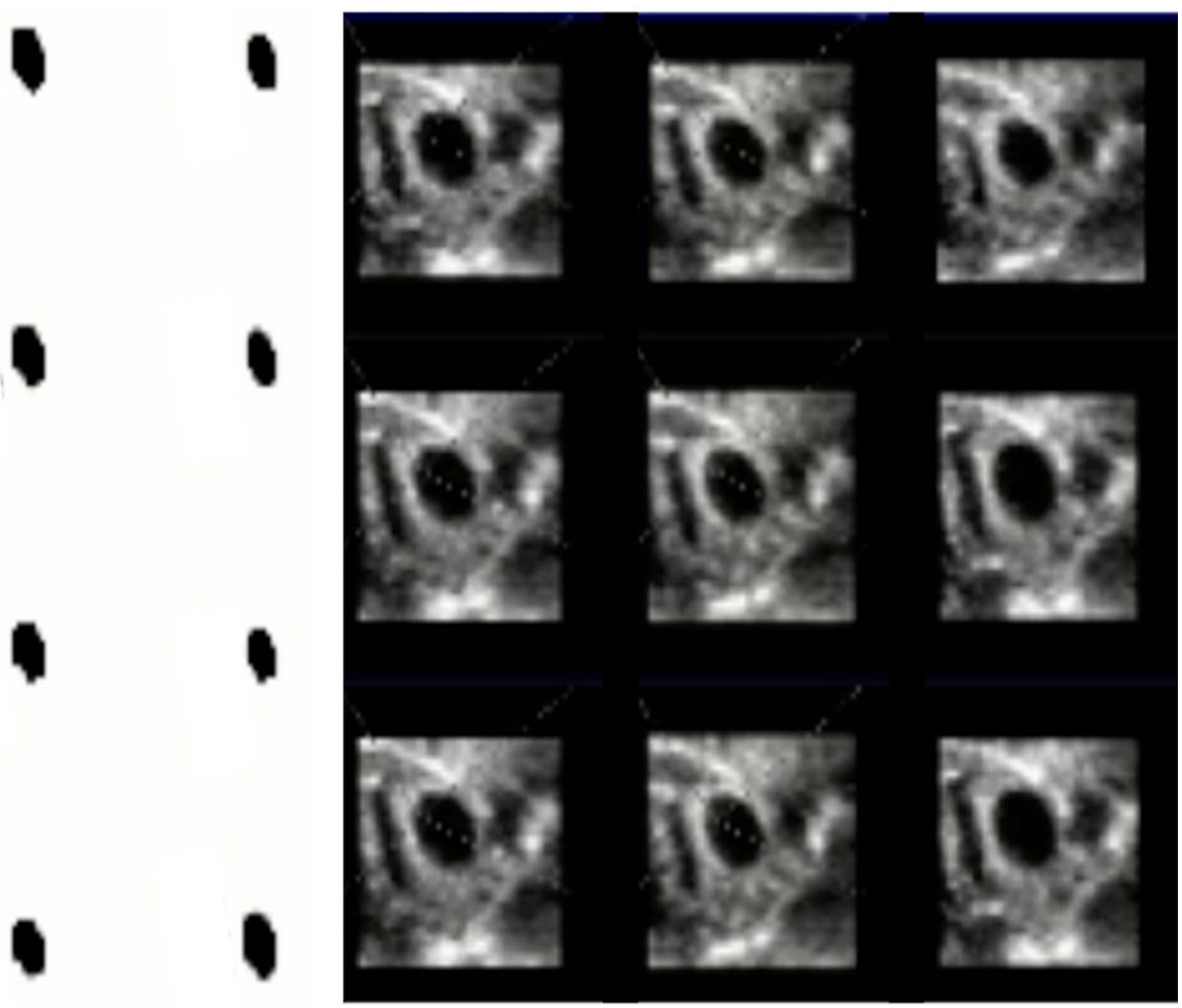

Figure 8: The final results for one case showing 9 LAA axial images as proposed landing zone 
An Algorithm for LAA Segmentation

that was higher than the calculated threshold value and hence loss of the object and failure in creating a binary image. Notably, the problem could be overcome by manual adjustment in Gaussian filter and gamma correction.

\section{Discussion}

In this study we have developed a simple and fast algorithm for semi-automated segmentation of a region of interest in the LAA so-called device landing zone.

Segmentation is a process that divides the image to separate regions so that each region is a collection of adjacent and interconnected pixels [11]. Segmentation is performed to parse an image into meaningful and easy to analyse regions. Some applications of medical image segmentation include determining the location of tumours and other injuries, measuring tissue sizes, and diagnosing illnesses. Numerous methods are in place for segmentation, including statistical, fuzzy clustering, optimization and graph-based methods. Statistical methods are very high performance, yet costly. Fuzzy clustering methods are also high performance, but their efficiency is much reduced for images with noise such as ultrasound echocardiography images. Optimization methods use any evolution algorithm, including genetic, particle swarm optimization, ant colony, tabu search, etc. to segment the image. Each of these methods have their own advantages and disadvantages [12]. However, there is a paucity of literature on the segmentation techniques on the echocardiographic images of the LAA.

\section{Conclusion}

In this study we used a simple algorithm for segmentation of the LAA device landing zone that might have important clinical implication. It is important to note that this study only demonstrates the feasibility of the algorithm in segmenting an area of interest in the LAA. The correlation of the average of perimeter derived diameter of the selected axial images as the landing zone, with the in vivo measure- ments and the chosen device is currently being studied.

\section{Conflict of Interest}

None

\section{References}

1. Arboix A, Alio J. Cardioembolic stroke: clinical features, specific cardiac disorders and prognosis. Curr Cardiol Rev. 2010;6(3):150-61. doi: $10.2174 / 157340310791658730 . \quad$ PMID: 21804774. PubMed PMCID: PMC2994107.

2. Kamel H, Healey JS. Cardioembolic Stroke. Circ Res. 2017;120(3):514-26. doi: 10.1161/CIRCRESAHA.116.308407. PMID: 28154101. PubMed PMCID: PMC5312810.

3. Kirchhof $P$, Benussi $S$, Kotecha D, Ahlsson A, Atar D, Casadei B, et al. 2016 ESC Guidelines for the management of atrial fibrillation developed in collaboration with EACTS. Eur Heart $\mathrm{J}$. 2016;37(38):2893-962. doi: 10.1093/eurheartj/ ehw210. PubMed PMID: 27567408.

4. Tzikas A, Holmes Jr DR, Gafoor S, Ruiz CE, Blomstrom-Lundqvist $\mathrm{C}$, Diener HC, et al. Percutaneous left atrial appendage occlusion: the Munich consensus document on definitions, endpoints, and data collection requirements for clinical studies. Europace. 2017;19(1):4-15. doi: 10.1093/europace/euw141.

5. Bhat M. Digital-Image-Processing. International Journal of Scientific and Technology Research. 2014;3(1):272-6.

6. Lee JS. Digital image smoothing and the sigma filter. Computer Vision, Graphics, and Image Processing. 1983;24(2):255-69. doi: 10.1016/0734189X(83)90047-6.

7. Horiuchi T, Watanabe K, Tominaga S, editors. Adaptive filtering for color image sharpening and denoising. 14th International Conference of Image Analysis and Processing-Workshops (ICIAPW 2007); IEEE; 2007. doi: 10.1109/ICIAPW.2007.11.

8. Senthilkumaran N, Rajesh R. Edge Detection Techniques for Image Segmentation - A Survey of Soft Computing Approaches. International Journal of Recent Trends in Engineering. 2009:1:2504. doi: 10.1109/ARTCom.2009.219.

9. Zaitoun NM, Aqel MJ. Survey on Image Segmentation Techniques. Procedia Computer Science. 2015;65:797-806. doi: 10.1016/j. procs.2015.09.027.

10. Sezgin M, Sankur B. Survey over image thresh- 
Pakizeh Moghadam A. et al

olding techniques and quantitative performance evaluation. Journal of Electronic Imaging. 2004;13(1):146-65, 20. doi: 10.1117/1.1631315.

11. Petrou M, Petrou C. Image Segmentation and Edge Detection. Image Processing: The Fundamentals, Second Edition. Wiley \& Sons, Ltd;

2011. p. 527-668.

12. Maleki I, Ghaffari A, Masdari M. A New Approach for Software Cost Estimation with Hybrid Genetic Algorithm and Ant Colony Optimization. International Journal of Innovation and Applied Studies. 2014;5(1):72-81. 